\title{
Usages de psychoactifs, rôles sexuels et genre en contexte festif gay (Paris/Toulouse, 2007)
}

Psychoactive substance consumption, gender roles and sexual practices in gay socializing (Paris/Toulouse, 2007)

\section{Sandrine Fournier}

\section{(2) OpenEdition \\ Journals}

Édition électronique

URL : http://journals.openedition.org/clio/9648

DOI : 10.4000/clio.9648

ISSN : 1777-5299

Éditeur

Belin

Édition imprimée

Date de publication : 1 mai 2010

Pagination : 169-184

ISSN : 1252-7017

Référence électronique

Sandrine Fournier, "Usages de psychoactifs, rôles sexuels et genre en contexte festif gay (Paris/ Toulouse, 2007) », Clio. Femmes, Genre, Histoire [En ligne], 31 | 2010, mis en ligne le 28 mai 2010, consulté le 19 avril 2019. URL : http://journals.openedition.org/clio/9648; DOI : 10.4000/clio.9648 


\title{
Usages de psychoactifs, rôles sexuels et genre en contexte festif gay (Paris/Toulouse, 2007)
}

\author{
Sandrine FOURNIER
}

"On sort, on danse, on prend des trucs, on rentre, on baise ». La consommation de substances psychoactives est, en 2007, partie intégrante de certaines soirées gay et l'articulation entre contexte festif, consommation de psychoactifs et sexualité, ainsi résumée par ce clubber parisien, semble d'évidence. L'usage de substances licites ou illicites répond à différentes fonctions dans le contexte de certaines formes institutionnalisées de la sociabilité gay (bars, clubs, backroom ${ }^{1}$, etc.) et plus largement dans celui de la sexualité furtive ${ }^{2}$. Par ses effets attendus sur les inhibitions, il facilite la rencontre de partenaires occasionnels, stimule le désir, augmente le plaisir sexuel et la performance, aide à la pénétration anale, accompagne la découverte de nouvelles pratiques et l'actualisation des fantasmes, etc.

1 Ce terme anglais (arrière-salle) désigne un espace situé à l'intérieur d'un bar ou d'un club gay dédié à l'activité sexuelle. Il est communément retraduit en français par le terme "bordel".

2 Voir sur cette question l'article de Myers et al. 2004 aux États-Unis, ainsi que celui de Green \& Halkitis 2006, portant plus spécifiquement sur l'usage du Crystal. 
Cet article s'appuie sur une enquête ethnographique 3 portant sur l'usage de substances psychoactives en contextes festifs gay à Paris et à Toulouse en 20074. Le réseau TREND (Tendances Récentes et Nouvelles Drogues) de L'Observatoire Français des Drogues et des Toxicomanies a commandé cette enquête en 2006 après avoir fait le constat que des produits spécifiques (GHB/GBL et kétamine ${ }^{5}$ ) étaient utilisés en club dans ce groupe, provoquant de nombreux comas dus au surdosage de ces substances. Par ailleurs, la presse gay faisait état de rumeurs autour de la diffusion du Crystal, une méthamphétamine réputée très addictive et dont l'usage favoriserait les comportements sexuels à risque selon certaines études ${ }^{6}$. Les résultats des études internationales établissant une cooccurrence entre prise de psychoactifs et prise de risques sexuels s'avèrent néanmoins contradictoires ${ }^{7}$. La quasi-absence de données qualitatives sur cette question, comme sur celle des consommations en contextes festifs gay français, a motivé la réalisation de cette première étude exploratoire.

3 La méthode d'enquête inclut des observations participantes en contextes festifs et des entretiens ouverts et semi-directifs avec trente-cinq usagers de substances psychoactives et quinze professionnels et bénévoles œuvrant dans le champ de la prévention contre le VIH et/ou la toxicomanie (informateurs clefs). Les usagers âgés de vingt-deux à quarante-cinq ans, majoritairement français (trente-quatre) et socialement intégrés, s’identifient comme gay. Quinze d'entre eux déclarent être séropositifs au VIH. Le recrutement des usagers de psychoactifs s'est déroulé suivant la méthode dite "boule-de-neige", à partir de réseaux de connaissance de la chercheuse, rendant ainsi possible l'accès à une population cachée.

4 Fournier \& Escots 2010.

5 Ces deux anesthésiques, classés comme stupéfiants, sont détournés de leur usage pour leurs propriétés relaxantes, euphorisantes, dissociatives, aphrodisiaques, etc. L'étude montre que la consommation de ces deux produits en contexte festif gay est étroitement associée à une "intentionnalité" sexuelle, dans la perspective de l'activité sexuelle qui suit la fête. La kétamine est plus spécifiquement utilisée pour ses propriétés anesthésiantes dans les pratiques sexuelles dites “hard". Le GBL, un décapant industriel non classé comme stupéfiant, se transforme en GHB dans le corps au cours du processus de digestion.

6 Kurtz 2005.

7 Gillmore et al. 2002. 
L'étude s'est donc pour une part attachée à décrire et analyser l'articulation entre les usages de psychoactifs, les pratiques sexuelles et les comportements à risque. Les fonctions, les effets perçus et attendus, le choix (relatif) des produits, de leur association et des modes d'usage associés à l'activité sexuelle varient suivant les personnes, les contextes, les partenaires, les pratiques, et suivant le moment de la «carrière » sexuelle. On examinera ici un point particulier: la fonction assignée à certains usages associés à la pénétration anale, en tant qu'elle révèle, d'une part les normes de genre, d'autre part, les normes dominantes dans une culture érotique particulière. Au plan individuel, on verra combien des normes parfois contradictoires engendrent des tensions dans le processus de construction des identités sexuelles et sexuées.

\section{Une consommation sexuelle « libérée ", virile, performante}

Interroger les usages de substances psychoactives associés à la sexualité, c'est rencontrer des (sous) cultures érotiques à l'intérieur desquelles les énoncés ayant trait aux effets subjectifs des psychoactifs sur le désir, le plaisir ou la performance sexuelle font sens à un moment donné et dans un groupe particulier.

Commençons donc par caractériser le contexte idéologique référant en matière de sexualité parmi les clubbers gay interrogés en 20078. D'abord la valorisation d'un rapport «libéré » à la sexualité, incluant la capacité à dissocier activité sexuelle et sentiments, est un énoncé revendiqué par la majorité des informateurs. "C'est toute la différence, on s'encombre pas de tout l'apanage, de la procédure, du protocole, voilà, de séduction. (...) On a autre chose à faire. (...) On s'emmerde plus. T'en as envie, j'en ai envie, on y va » (Rodrigue, 42 ans). Une capacité construite comme étant de "nature» masculine, pouvant librement s'exercer entre hommes, comme le précise ce quadragénaire parisien lorsqu'on l'interroge sur la spécificité du festif gay: "même si j'avais envie, je vais jamais dire à une meuf: "Je te baiserais bien." Je vais me faire traiter de macho, et... Alors que là on est entre mecs, on peut, on a cette liberté de se dire: "Ie te baiserais bien" on "I'aurais bien envie de niquer" on "Tu veux pas que je te

8 Il s'agit bien d'un sous-groupe, le propos n'est pas généralisable à tous les hommes ayant des rapports sexuels avec des hommes, loin s'en faut. 
suce?". Et il y a rien de dégradant, il y a rien d'insultant, ce qui change complètement la donne. (...) Les mecs arrivent à faire la part des choses entre la relation avec le sentiment et le plan cul.»

La valorisation de la virilité est un autre élément récurrent. " $\mathrm{La}$ mode actuelle c'est: gonflette, Musclor, GHB'. On disait de certains pédés des années 90 qu'ils avaient le "look hétéro". On ne dit plus "look hétéro" parce que la virilité est la norme. On désigne plutôt les folles, les folasses. La mode des folles est passée » (Pedro, 36 ans).

"Le culte de la performance" n'est pas moins prégnant, directement et indirectement dans les discours sur la sexualité. Les représentations des corps et des pratiques sexuelles telles qu'elles sont véhiculées et mises en scène par certains films pornographiques renvoient à des stéréotypes sur la taille du sexe, sur l'hyper virilité et sur la capacité d'endurance au cours de l'acte sexuel. Ces référents deviennent normatifs dans certains contextes, notamment celui des rencontres et des échanges via Internet: "Mais c'est aussi en plus ce qu'on vébicule dans les sites gay, dans les pornos aussi. C'est que ça a l'air tellement simple d'un autre côté, quand tu vois les films pornos, quand tu vois comment ça chat. Tu te dis mais t'as vraiment affaire maintenant à des professionnels de la queue, quoi » (François, 32 ans).

Il semble qu'on soit passé de la consommation sexuelle à une offre par segments de marché dans un contexte où la spécialisation des pratiques est favorisée par le développement d'Internet et la profusion de l'offre (sites et soirées). Ainsi, Xavier compare la rencontre d'un homme sur Internet pour «un plan cul» à la commande d'une pizza : "Si j'ai demandé avec anchois, je veux ce que j'ai commandé et pas autre chose. "S'agissant de l'outil internet en tant que moyen de rencontres, on en valorise volontiers l'aspect fonctionnel : "Tu vas pas perdre trois heures à draguer, à payer des verres pour te rendre compte au final que vous êtes pas compatibles » (Pedro). Le développement d'Internet constitue un bouleversement majeur tant au niveau de l'apprentissage, des pratiques, des modèles référents que des modes

9 Ce triptyque désigne un groupe particulier, réputé consommateur de GHB, et qui est identifiable à son apparence virile et ses caractéristiques physiques (corps très musclés, travaillés dans les salles de musculation, tatoués et percés). 
de rencontre et de sociabilité ${ }^{10}$. Comme le constatent deux sociologues analysant l'impact de son usage sur l'évolution de la sexualité : «Échappant à la pression normative visant à la régulation sociale de la sexualité, le réseau est donc propice à l'expression de la diversité des sexualités entre hommes $»^{11}$.

\section{Psychotropes et consommation sexuelle}

Bien que la consommation sexuelle soit devenue la norme dans certains sous-groupes, la capacité à «consommer » du sexe dépend d'un ensemble complexe de facteurs tels que l'âge, l'état psychique, l'état affectif, la relation à la sexualité, l'expérience, etc. et peut varier pour une même personne au cours du temps.

Lors des soirées où la recherche d'un partenaire sexuel est un moteur de la fête, la consommation de nombreux produits (alcool inclus), facilite la rencontre de partenaires occasionnels. Elle renforce l'assurance, la confiance, l'estime de soi, annihile la peur du rejet, favorise l'expression du désir, en même temps qu'elle participe des manières d'être qui sont attendues sur un marché des rencontres particulièrement concurrentiel (être beau, en forme, performant, jovial, etc.). Sur ce terrain, on peut qualifier certaines logiques de consommation de conventionnelles, en ce sens qu'elles visent à une adaptation optimisée à l'univers de la fête ou de la sexualité furtive. Les normes prévalant dans certains sous-groupes dans ce contexte sont liées, sous différents rapports, aux normes en vigueur dans l'ensemble de la société. Ces conduites “dopantes", valorisées dans la fête comme dans la sexualité, sont cohérentes avec les comportements attendus ou encouragés dans d'autres sphères de la vie sociale, celle du travail par exemple. Ainsi, certains "butch"12, décrits par tous les informateurs comme constituant l'élite

10 Par rapport à son édition de 2000, l'Enquête Presse Gay 2004 constate la hausse de fréquentation de la toile, qui passe de $17,7 \%$ à $35,1 \%$, parallèlement à la baisse continue de la fréquentation des bars et des discothèques sans backroom (qui passe de $42,8 \%$ en 1997 à 32,1\% en 2004).

11 Léobon \& Frigault 2004.

12 Cette abréviation du mot anglais "butcher" (boucher), empruntée à la culture lesbienne, désigne un gay à l'apparence très masculine. 
économique des clubbers gay et réputés grands consommateurs de psychoactifs, occupent des emplois dans les domaines de la finance, du marketing, de la communication, bref des activités où l'esprit de compétition et le dépassement des limites individuelles sont fortement valorisés.

Dans le cadre de rencontres furtives - où «tu baises pas non plus qu'avec des mecs qui te plaisent vraiment» (Bertrand, 28 ans) -, les psychoactifs, en modifiant le rapport à soi et à autrui, favorisent l'excitation et le plaisir notamment avec un partenaire qui, autrement, ne susciterait pas de désir. L'altération du jugement sous l'effet des produits permet de se concentrer sur son désir en faisant abstraction de l'autre : " "Oh là, là, là, putain, la sale gueule!” ou "Putain, il pue de la gueule!" Tu vois des trucs finalement où quand t'es sous l'influence de tel stupéfiant, tu t'en fous et du coup tu vas aller vachement plus à l'essentiel, c'est-àdire prendre du plaisir avec lui » (François, 32 ans). L'usage de substances participe de la perception d'un autre "dissocié", dissociation de la personne et de son corps, dissociation des parties du corps. Il favorise une perception fragmentaire du corps de l'autre : «(...) Sous produit, le mec, en fait, je vais pas regarder vraiment s'il me plaît ou pas je vais surtout regarder son cul, sa bite. En fait, je vais faire la crevette. Je vais enlever la tête, je vais regarder son corps » (Karl, 30 ans).

\section{Les psychoactifs et la sexualité "active"}

Il est apparu au cours de l'enquête qu'il semblait y avoir une différenciation de la consommation des substances psychoactives - ad minima d'un point de vue quantitatif-suivant le rôle sexuel que l'informateur déclarait endosser préférentiellement.

Les produits les plus directement associés à la pénétration anale insertive ${ }^{13}$ sont les médicaments de la performance sexuelle (Viagra ${ }^{\circledR}$, Cialis ${ }^{\circledR}$ et leurs génériques). Bien que ceux-ci n’aient aucune propriété psychoactive, ils peuvent être utilisés marginalement à des fins proches de l'usage de drogue, dans la mesure où l'altération physiologique attendue (érection, virilité et agressivité associées) modifie le rapport à soi et à autrui. On observe que certains en font

13 La pénétration anale insertive désigne le rôle sexuel de celui qui pénètre. La pénétration anale réceptive désigne le rôle sexuel de celui qui est pénétré. 
usage régulièrement ou systématiquement lors de rencontres furtives, où comme l'explique Édouard, l'injonction d'être performant est particulièrement prégnante: "J'en prends quasiment tout le temps maintenant. (...) Étant plus souvent actif, il y a une énorme pression qui fait que, le problème, c'est que t'as pas le droit à l'erreur maintenant et pour débander il y a rien de pire. C'est-à-dire qu'avant il y avait une certaine... On disait, bon, tu bandes à $90 \%$, bon ben, c'est pas grave. Maintenant si tu bandes pas à 100\% dur, machin, y a aucune... T'es jeté de toute façon. On te dit: "Bon allez, casses-toi!" en 2 secondes, c'est vite vu. Et voilà, et ça, c'est le truc le plus bloquant pour un actif et qui fait que automatiquement, tu anticipes cette peur-là et déjà avant, tu bandes pas de toute façon. Donc maintenant, quand j'ai vu ça, j'en prends même un quart ou un dixième, déjà pour avoir cet effet placebo et déjà tu enlèves cette angoisse. »

S'agissant de l'effet des produits psychoactifs associés à l'acte de pénétration insertive, l'idée que leur consommation peut contrarier la capacité érectile est très souvent évoquée. Les effets supposés des substances peuvent amener les usagers ayant une préférence pour le rôle sexuel actif, soit à dissocier dans le temps consommation et sexualité, soit à limiter la consommation, soit, depuis quelques années, à soutenir l'activité sexuelle avec les médicaments de la performance sexuelle. Pour d'autres, l'effet perçu sur la capacité érectile ne pose pas de problème puisqu'ils déclarent attendre des effets des produits un autre rapport à la sexualité, affranchi des contraintes de la sexualité ordinaire. La représentation généralement admise est que le comportement actif est d'autant mieux exercé que l'homme est en bonne forme physique, reposé et à jeun. Il s'agit de maîtriser la "machine" corporelle. La sexualité de l'actif est affaire de corps : "(...) Il y a aussi un côté mécanique. C'est-à-dire que si tu prends trop de coke ou trop de poppers on trop de... tu peux plus être que passif, t'as plus le choix (rires)" (Bertrand). "De toute façon quand tu es actif, il faut que tu maîtrises un certain nombre de choses. Déjà il faut que tu contrôles, pas jouir trop vite, pas rester non plus trop longtemps sans jouir» (Pedro). La description des effets des médicaments de la performance sexuelle est ainsi décrite comme étant strictement d'ordre mécanique. Les substances agissent sur le corps de l'actif, tandis qu'elles sont réputées agir au plan psychique sur le passif: "Toute drogue fait perdre ses moyens, dilate, pas mécaniquement mais au moins mentalement l'anus» (Pierre, 24 ans). On 
note ainsi le parallèle avec le préjugé commun situant la sexualité dans le corps des hommes (actifs) et dans la tête des femmes (passives). Plus généralement, on rencontre, au travers des représentations des effets des produits associés à l'activité sexuelle, l'expression d'une équivalence symbolique entre comportements/rôles sexuels et identités sexuées qui renvoie, nous allons le montrer, aux représentations communes de la sexualité des hommes et des femmes.

\section{Les psychoactifs et la sexualité "passive"}

Lors des premiers entretiens, les informateurs évoquent d'abord les effets vasodilatateurs et relaxants de certains produits (les poppers ${ }^{14}$ par exemple), dans la pénétration anale réceptive tandis que le rôle actif nécessiterait, comme on l'a vu, une gestion particulière de la consommation. Lors d'entretiens réalisés ultérieurement, la question des effets perçus des produits, avant ou pendant l'acte sexuel, est explorée plus précisément. On constate alors que toute substance, quels que soient ses effets perçus, semble convenir à la pénétration anale réceptive. De plus, certains déclarent désirer être pénétrés et adopter ce rôle exclusivement sous l'effet de substances psychoactives. Au-delà de l'effet "mécanique" vasodilatateur et musculo-relaxant, quelque chose d'autre semble donc jouer dans la pénétration anale réceptive et nous amène à nous interroger sur les représentations des rôles sexuels dans ce groupe.

"C'est rare que les gens affirment: "Je suis super passive!" C'est rare parce qu'il y a toujours une connotation de "Ah! C'est une folle, c'est pas un vrai mec” (Informateur clef). La féminisation de l'adjectif ou du substantif désignant celui qui est pénétré est fréquente, souvent

14 Préparation conditionnée à l'état liquide, les poppers sont détournés de leur usage (cardiologique) pour leurs propriétés vasodilatatrices et pour la brève euphorie qu'ils provoquent lorsqu'ils sont inhalés. Ce produit est, de loin, le psychoactif le plus consommé parmi les gay depuis de nombreuses années. Ses effets attendus sur la sexualité sont multiples : il augmente l'excitation et le plaisir sexuels; il permet de retarder l'éjaculation, d'amplifier les sensations orgasmiques, et surtout, de l'avis de tous les informateurs, il «aide», par ses effets vasodilatateurs, la pénétration réceptive. 
associé à un superlatif ("C'est une grosse passive!»). De même, la féminisation des organes génitaux de l'homme qui est pénétré n'est pas rare; le mot «chatte » désignera par exemple les fesses au cours de l'acte sexuel. On observe encore, au travers des discours, l'association entre rôles sexuels (pénétrant/pénétré), comportements sexuels (actif/passif) et les identités masculines et féminines. Cette superposition des comportements sexuels et des identités de genre semble charrier les représentations et les valeurs différentielles qui leur sont associées: "Même chez les pédés entre eux. On va entrer dans un bordel (backroom), on va voir un beau gosse, il va se taper trois-quatre mecs, on va dire: "Putain le bean gosse, il s'en est tapé trois-quatre!" On voit un mec avec le cul à l'air qui vient de se faire baiser trois-quatre fois, on aura pas du tout la même image: "Ah! La salope, putain, il me dégô̂te"»(Rodrigue). On retrouve bien les archétypes du «Don Juan » et de la «salope » et plus généralement la référence aux rapports hétérosexuels tels qu'ils sont communément représentés : "Moi je suis tombé sur beaucoup d'actifs qui n'en avaient rien à faire de savoir si j'avais du plaisir ou pas» (Pierre).

Lors des rencontres furtives, l'injonction d'un rapport "libéré" à la sexualité peut être diversement assumée suivant le rôle sexuel dans une interaction donnée. Pour certains hommes, tenir un rôle passif n'est pas si aisé : "Il y a une sous-déclaration des passifs 》 (Anatole, 31 ans). "Dans la réalité, on pourrait dire qu'il y a les actifs, les passifs et les gens qui se prétendent actifs » (Paul, 41 ans). Chez certains, assumer ce rôle se traduit par un jeu d'équilibre entre présentation de soi et pratiques sexuelles: "C'est très marrant parce que mon ex, lui, se pare de tous les attributs masculins. Ca veut dire qu'il s'habille avec des treillis et, qu'au fond, il y a ça qui est très prégnant chez. lui, il est très passif » (Antonin, 32 ans).

C'est en réalisant un entretien avec un informateur ayant commencé à vivre "tardivement" des relations avec des hommes que l'on a eu accès aux énoncés les plus explicites de l'équivalence entre d'une part le passif et le féminin et d'autre part l'actif et le masculin. Dans ce cas, le processus de déconstruction de l'identité (hétéro)sexuelle et, parallèlement, l'apprentissage de l'homosexualité ont sans doute favorisé la réflexivité sur cette question. Xavier, trentenaire célibataire, vit des relations furtives avec des hommes rencontrés sur Internet, en inhalant systématiquement des poppers. Dans cette phase d'apprentissage, il explique comment il ressent le 
fait d'être pénétré : "Forcément le fait d'être passif, t'es complètement offert à l'autre et, à un moment, t'es pas complètement offert à l'autre mais en tout cas t'es dans une phase de vulnérabilité. (...) Pour moi c'est vraiment un rapport de domination et tout va se jouer dans ce rapport de domination. Et je sais très bien que, moi, quand je suis passif avec des mecs, et là dernièrement c'est vrai que j'étais beaucoup plus passif qu'actif, le rapport se joue différemment. Les mecs se sentent des vrais mecs. Autant, on a l'impression que tu es simplement le trou de service. » On voit bien ici la manière dont est ressentie cette double équivalence symbolique entre passivité/féminité et activité/masculinité, associée, dans ce cas particulier, aux postures dominant/dominé. Lorsque, au cours de l'entretien, est abordée la question de l'apport de l'usage de produit dans la sexualité, loin de mettre en avant les effets vasodilatateurs des poppers, Xavier énonce deux raisons : "Tu enlèves tout le carcan social de ce qu'est un mec: c'est lui qui baise, c'est pas lui qui se fait baiser. (...) Et puis, il y a ce côté de se dire, c'est pas vraiment moi. Dans la vie de tous les jours, je suis pas comme ça, par contre sous produits, je peux être comme ça.» Pour lui, c'est bien l'identification du rôle (pénétré) à la féminité qui semble motiver l'usage d'une substance psychoactive qui, au-delà de ses effets sur l'excitation et le plaisir, lui permet de vivre l'acte parce que, sous l'influence du produit, il n'est pas tout à fait lui-même. En tant qu'il est un autre, assumer un désir socialement construit comme féminin ne perturbe pas son identité masculine.

Se déprendre des constructions sociales associées à celui qui est pénétré a requis, pour la majorité des informateurs, un apprentissage ${ }^{15}$. Tous évoquent la douleur ressentie pendant une période plus ou moins longue. Tous n'ont pas eu recours à des produits pour ressentir du plaisir dans cet acte. On constate plus généralement au cours des entretiens que la relation anale a souvent lieu, avec ou sans produit, entre partenaires réguliers entre lesquels une relation de confiance est établie. Dans le cadre de relations stables, plusieurs informateurs ayant exclusivement un comportement sexuel actif avec leur partenaire font état de leurs difficultés à être

15 Voir, sur la question du sexe anal et de la catégorisation sociale de cette pratique dans différentes cultures, l'article de Mendes-Leité 2003; sur les enjeux de pouvoir associés à la pénétration voir Kippax \& Smith 2001. 
pénétrés et, dans ce cas, l'usage de substances psychoactives peut être la condition nécessaire du désir et de l'actualisation de cette pratique : "Sur la sodomie (...) tu vas te laisser plus aller, donc du coup, tu vas peut-être plus facilement te laisser prendre » (François).

$\mathrm{Au}$ cours des relations furtives, la pénétration est le plus souvent associée à la consommation de psychoactifs : "Je pense que, bien sûr, les pratiques sont aussi liées à l'usage de drogue. Moi je serais plutôt passif quand je prends des ecstas ${ }^{16}$. Du moins, j'ai plus de facilité à l'être. (...) Je stresse moins, je suis byper cool. Alors que je dirais, à jeun, je me fais baiser, c'est pas ça, c'est clair. (...) Ca induit, je me dis les drogues ça vient modifier la perception qu'on a. Je pense aussi que ça déverrouille dans la tête. (...) Un truc qui fait qu'à un moment je suis plus à l'aise donc les choses se passent mieux. Mais que je n'ai pas la capacité de déverrouiller quand je suis, je dirais, réellement moi-même» (Rodrigue).

Parce que les effets attendus de la consommation de substances psychoactives ont trait à leur action supposée sur les inhibitions, l'usage qui en est fait dans les relations sexuelles est, selon nous, un bon révélateur de l'imprégnation des règles sociales assignant à chaque sexe le code de conduite approprié dans l'acte sexuel autant que de l'idéologie normative de référence dans un sous-groupe particulier. «Être un autre» sous leur influence peut autoriser à s'affranchir de certaines normes ou aider à en actualiser d'autres.

On constate, dans tous les cas, que les énoncés où il est question de l'usage de produits psychoactifs dans les relations sexuelles renvoient implicitement ou explicitement à la question des normes. Plus généralement, la consommation "autorise" l'actualisation de fantasmes ou la découverte de nouvelles pratiques contrevenant aux prescriptions sociales en matière de genre ou de sexualité : «ça t'aide à te... à te libérer plus, à pas mettre trop de barrières. Pas avoir de moralité dans la sexualité » (Sofiane). "L'autre sous influence » peut alors jouer (ou sur-

16 L'ecsta, aussi nommé $X$ ou tase, abréviations d'ecstasy, désigne la forme comprimée de la MDMA, une substance de synthèse appartenant à la famille des amphétaminiques. C'est un psychostimulant ayant des effets euphorisants et empathogènes ("qui rendent love »). Ce produit est fréquemment consommé dans différents contextes festifs où est diffusée de la musique électronique depuis les années 1990. Réputé augmenter les sensations tactiles, il est plus particulièrement associé à l'activité sexuelle chez les gay. 
jouer) avec un ensemble d'oppositions symboliques sans craindre le regard ou le jugement du tiers : féminin/masculin ("Tu vas t'inventer aussi un personnage d'un mec byper macho, ou, à la limite, d'un mec byper féminin où là tu vas vraiment jouir comme une femme grâce à cette drogue-là »), humain/animal ("Si tu veux être une bonne chienne, tu prends du GHB»), dominant/dominé ("S'abandonner totalement, être une merde, être l'objet sexuel de l'autre »), etc. Si ces jeux d'oppositions traduisent, pour une part, les représentations sociales et les classifications hiérarchisées qui les sous-tendent, plaçant du même côté le féminin, l'animal, le dominé, elles n'en traduisent pas moins la possibilité offerte de jouer avec les normes de sexe et de genre et, ce faisant, de contribuer à les "dénaturaliser". On peut en effet reprendre ici l'analyse de Judith Butler sur le désir lesbien pour voir que «l'identification » avec le féminin du rôle sexuel passif n'est pas de l'ordre d'une pure assimilation de l'homme au féminin: "C'est la déstabilisation du rapport entre le corps et l'identité, le féminin et le masculin qui devient érotique ${ }^{17}$. Notons que ces jeux d'inversions sont rendus possibles dans un contexte culturel caractérisé, d'une part, par la coexistence de différentes normes sexuelles ${ }^{18}$, d'autre part, par la (relative) dissociation pensable entre sexe, genre et sexualité. En effet, l'anthropologie nous montre que, dans nombre de sociétés, les pratiques sexuelles plus ou moins institutionnalisées et/ou socialement acceptables entre hommes s'accompagnent d'une claire distinction de genre. Celui qui est pénétré endosse alors les attributs dévolus aux femmes (vêtements, comportements, tâches domestiques, etc.). Le modèle «hétérosocial» est ainsi respecté19. Si, comme on l'a vu, l'assignation sociale de la pénétration réceptive au féminin reste prégnante dans nos sociétés, il n'en demeure pas moins que l'action des mouvements féministes, puis des mouvements gay a contribué à une remise en cause de ces catégorisations et permis, au plan individuel, un désapprentissage des normes incorporées, parfois à l'aide de psychoactifs.

17 Butler $2006: 241$.

18 Gagnon 2008.

19 Mathieu 2000. Sur la question de la relation entre homosexualité et efféminement, voir également l'article de Prieur 1998. 
La consommation de substances psychoactives associée à la fête et à la sexualité n'est certainement pas propre aux gay. Pour l'anthropologue Alain Ehrenberg, les usages de drogue constituent aujourd'hui «des expériences qui produisent et révèlent simultanément les styles de rapports que l'individu entretient avec lui-même et avec autrui $»^{20}$, traduisant ainsi un mode existentiel propre aux sociétés occidentales contemporaines. Joseph L. Lévy rappelle, dans son introduction au dossier de la revue Drogue, santé et société consacré aux usages de psychotropes associés à la sexualité, que «l'une des fonctions importantes associées à l'usage des drogues et de l'alcool renvoie à la régulation de l'expression de la sexualité, et ce, tant dans les sociétés traditionnelles que dans les sociétés moderne $»^{21}$. L'action supposée ou réelle des plantes et des produits sur le désir, la sensibilité corporelle, l'excitation, le plaisir ou la réduction des inhibitions est ancienne et n'en suscite pas moins l'intérêt de nos contemporains. De même, les médicaments dont on a découvert les effets sur la sexualité ont intégré le lot des drogues illicites récréatives. Ce phénomène a été encore renforcé à partir des années 1980, lorsque la recherche pharmaceutique s'est orientée plus directement vers le traitement des dysfonctions sexuelles masculines ${ }^{22}$. En outre, des femmes hétérosexuelles utilisent aussi des substances psychoactives comme l'ecstasy pour ses effets sur les inhibitions, dans le but de découvrir de nouvelles pratiques ou d'augmenter les sensations orgasmiques ${ }^{23}$.

Ainsi, au-delà de la spécificité des pratiques sexuelles gay, on voit bien, qu'au travers des discours qui en rendent compte, on rencontre des énoncés articulant l'usage avec un ensemble de catégories qui sont sous-jacentes dans tout type de sexualité. Si les usages rencontrés dans un groupe particulier font sens au-delà des contextes spécifiques dans lesquels ils sont observés, alors il convient de dé-spécifier ces énoncés. Si on considère que l'expérience sexuelle est un lieu central

\footnotetext{
20 Ehrenberg 1991: 6.

21 Lévy $2006: 5$.

22 Lévy \& Garnier 2006.

23 Garceau-Broder 2006.
} 
de la construction morale de soi en Occident ${ }^{24}$, on entrevoit l'intérêt d'étendre l'examen des rôles, des sens, des fonctions, des représentations et des croyances assignés aux produits psychoactifs quand ils sont associés à la sexualité à d'autres cultures érotiques pour analyser la construction des identités sexuelles et sexuées des hommes et des femmes au travers de l'activité sexuelle.

\section{Bibliographie}

ANRS, INVS, 2007, Rapport enquête Presse Gay 2004, Saint-Maurice (Fra), Institut de veille sanitaire.

BOZON Michel, 2001, "Orientations intimes et construction de soi. Pluralité et divergences dans les expressions de la sexualité », Sociétés contemporaines, numéro thématique «Les cadres sociaux de la sexualité », 41-42, p. 11-40.

BuTLER Judith, 2006, Trouble dans le genre (1 ${ }^{\mathrm{re}}$ édition américaine 1990), traduit par Cynthia Kraus, Paris, La Découverte.

EhreNBerg Alain (éd.), 1991, Individus sous influence: drogues, alcools, médicaments psychotropes, Paris, Éditions Esprit.

FOURNIER Sandrine \& Serge ESCOTS (éd.), 2010 (à paraitre), Homosexualité masculine et usage de substances psychoactives en contextes festifs gais. Enquête ethnographique à Paris et Toulouse en 2007, Saint-Denis, OFDT.

GAGNON John, 2008, Les scripts de la sexualité. Essais sur les origines culturelles du désir, traduit par Marie-Hélène Bourcier avec Alain Giami, Paris, Payot.

Garceau-Broder Marie-Hélène, 2006, «Ecstasy et sexualité : une étude exploratoire au Québec », Drogue, santé et société, numéro thématique "Drogues et sexualité » dirigé par Joseph J. Lévy, vol. 2, 5, p. 111-134.

Gillmore Mary Rogers, Morrison Diane M., Leigh Barbara C., Hoppe Marilyn J., GAYLORD Jan, RaINEY Damian T., 2002, "Does "High = High Risk"? An Event-Based Analysis of the Relationship Between Substance Use and Unprotected Anal Sex Among Gay and Bisexual Men », Aids and Behavior, vol. 6, 4, p. 361-370.

Green Adam Isaiah \& Perry N. Halkitis, 2006, «Crystal methamphetamine and sexual sociality in an urban gay subculture: an elective affinity Culture, bealth \& sexuality", vol 8, 4, p. 317-333.

24 Bozon 2001. 
Kippax Susan \& Gary Smith, 2001, «Anal Intercourse and Power in Sex Between Men », Sexualities, vol. 4, 4, p. 413-434.

KURTZ Steven P., 2005, « Post-circuit blues: motivations and consequences of crystal meth use among gay men in Miami », Aides and Behaviour, 9, 1, p. 63-72.

LÉOBON Alain \& Louis-Robert FRIGAUlT, 2004 (décembre), Les usages sociosexuels d'Internet dans la population homo et bisexuelle française : résultats de l'enquête Net Gay Baromètre, Paris, Rapport de recherche ANRS. http://www.gaystudies.org

LÉVY Joseph J. (dir.), 2006, « Drogues et sexualité », numéro thématique, Drogue, santé et société, vol. 2, 5.

LÉVY Joseph J. \& Catherine GARNIER, 2006, «Drogues, médicaments et sexualité », Drogue, santé et société, vol. 2, 5, p. 11-47.

MAthiEu Nicole-Claude, 2000, "Anthropologie et homosexualités », in Martine Gross (dir.), Homoparentalités, état des lieux: parentés et différence des sexes, Issy-lesMoulineaux, ESF, p. 89-94.

MENDES-Leité Rommel, 2003, «Sens et contexte dans les recherches sur les (homo) sexualités et le sida : réflexions sur le sexe anal ", in Christophe BROQUA, France Lert, Yves Souteyrand (éd.), Homosexualités au temps du sida. Tensions sociales et identitaires, Paris, CRIPS, ANRS, p. 199-220.

Myers Ted, Aguinaldo Jeffrey P., Dakers D., Fischer Benedikt, Bullock Sandra, Millson Peggy \& Liviana Calzavara, 2004, « How drug using men who have sex with men account for substance use during sexual behaviors: questioning assumptions of HIV prevention and research », Addiction Research and Theory, vol. 12, 3, p. 213-229.

PRIEUR Annick, 1998, "Little boys in mother's wardrobe. Sur les origines de l'homosexualité et de l'efféminement. ", Actes de la recherche en sciences sociales, 125, p. 15-29. 